\title{
Generating Sharp Features on Non-regular Triangular Meshes
}

\author{
Tetsuo Oya ${ }^{1,2}$, Shinji Seo ${ }^{1}$, and Masatake Higashi ${ }^{1}$ \\ 1 Toyota Technological Institute, Nagoya, Japan \\ 2 The University of Tokyo, Institute of Industrial Science, Tokyo, Japan \\ \{oya, sd04045, higashi\}@toyota-ti.ac.jp
}

\begin{abstract}
This paper presents a method to create sharp features such as creases or corners on non-regular triangular meshes. To represent sharp features on a triangular spline surface we have studied a method that enables designers to control the sharpness of the feature parametrically. Extended meshes are placed to make parallelograms, and then we have an extended vertex which is used to compute control points for a triangular Bézier patch. This extended vertex expressed with a parameter enables designers to change the shape of the sharp features. The former method we presented deals with regular meshes, however, it can be a strong restriction against the actual variety of meshes. Therefore, we developed a method to express sharp features around an extraordinary vertex. In this paper, we present algorithms to express creases and corners for a triangular mesh including extraordinary vertices.
\end{abstract}

\section{Introduction}

Computer-aided design tools have supported the designer's work to create aesthetic and complex shapes. However, to represent a pleasing and high quality surface is still a difficult task. The reason of this difficulty is that both high continuity of the surface and the ability to handle 2-manifold surfaces with arbitrary topology are required especially in the industrial design field. In addition, sharp features such as creases and corners which play a significant role to express product's shape should be treated as they want. However, expressing sharp features at an arbitrary place is not easy. Therefore, a method to represent sharp features and to control their shapes would be a great help to designers.

There are two major ways to represent surfaces in computer graphics. In CAD/CAM software, tensor product surfaces such as Bézier, B-spline and NURBS are usually used to represent free-form surfaces. These are expressed in parametric form and generally have high differentiability enough to represent class-A surfaces. However, connecting multi patches with high continuity is rather difficult. Also, techniques like trim or blend are usually utilized to represent the required complexity, and it would be an exhausting job. Furthermore, it is difficult to generate sharp features on arbitrary edges.

The other important method, namely, subdivision surfaces have become a popular method recent years especially in the entertainment industry. Inputting

M. Bubak et al. (Eds.): ICCS 2008, Part II, LNCS 5102, pp. 66 75, 2008.

(C) Springer-Verlag Berlin Heidelberg 2008 
an original mesh, some subdivision scheme is repeatedly performed on the vertices and the faces of the mesh, then a refined resultant is obtained. Although its limit surface is theoretically continuous everywhere, the obtained surface is a piecewise smooth surface. Thus it is not applicable to the surfaces used in industrial design where high quality surfaces are always required. Moreover, the parametric form of the surface is not available.

As for sharp features on a subdivision surface, there are many studies dealing with creases and corners. Nasri [1] presented subdivision methods to represent boundary curves, to interpolate data points, and to obtain intersection curves of subdivision surfaces. Hoppe et al. 2] proposed a method to reconstruct piecewise smooth surfaces from scattered points. They introduced a representation technique of sharp features based on the Loop's subdivision scheme [3]. To model features like creases, corners and darts, several new masks were defined on regular and non-regular meshes. DeRose et al. 4] described several effective subdivision techniques to be used in the character animation. They introduced a method to generate semi-sharp creases whose sharpness can be controlled by a parameter. Biermann et al. 5] improved subdivision rules so as to solve the problems of extraordinary boundary vertices and concave corners. Based on this method, then Ying and Zorin [6] presented a nonmanifold subdivision scheme to represent a surface where different patches interpolates a common edge along with the same tangent plane. Sederberg et al. 7] presented a new spline scheme, called T-NURCCs, by generalizing B-spline surfaces and Catmull-Clark surfaces. In this method, features are created by inserting local knots. To create and change features, direct manipulation of knots and control points is required. These methods have succeeded to represent sharp features, however, the subdivision surface technique is not a principal method in an industrial design field where high quality smooth surfaces are demanded.

An alternative method to create surfaces is generating a spline surface composed of Bézier patches. Triangular Bézier patching can be used to represent complex models because each patches are easily computed from the original mesh. The advantage of this method is that it is rather easier to keep continuity across the patches than conventional tensor product patches. Hahmann [8] 9] has shown the effectiveness of spline surface technique. Yvart and Hahmann [10] proposed a hierarchical spline method to represent smooth models on arbitrary topology meshes. With their method, designers are able to create complex models preserving tangent plane continuity when refining a local patch to add details. However, they have not mentioned how to represent sharp features. Thus, to be a more practical method, representing sharp features on a triangular spline surface should be studied. Loop [11] represented a sharp edge as a boundary curve by connecting two patches, however, its shape is not controllable because it is depend on each patches' boundary curves. Higashi 12 presented a method to express sharp features by using the concept of extended mesh. With that method, the shape of the edge can be changed parametrically.

In spite of its high potential, the triangular spline technique is not frequently used like other methods. One of the reasons is its difficulty of handling non-regular meshes. Here, a non-regular mesh means a mesh containing an extraordinary 
vertex whose valence is not six. In this paper, we developed a method to represent controllable sharp features on a non-regular triangular mesh.

This paper is organized as follows. Sec. 2 presents basics on Bézier representation used in this paper. Sec. 3 describes the method of mesh extension to express sharp features. In Sec. 4, we present main contributions of this paper, that is, schemes to handle non-regular meshes. Then several examples are shown in Sec. 5 , and Sec. 6 concludes this paper.

\section{Triangular Bézier Patch}

To construct a triangular spline surface, we utilize a triangular Bézier patch. In this section, we briefly describe important backgrounds about Bézier forms 13 .

A Bézier surface of degree $m$ by $n$ is defined as a tensor product surface

$$
\mathbf{b}^{m, n}(u, v)=\sum_{i=0}^{m} \sum_{j=0}^{n} \mathbf{b}_{i, j} B_{i}^{m}(u) B_{j}^{n}(v)
$$

where $\mathbf{b}_{i, j}$ is the control net of a Bézier surface, and $B_{i}^{m}(u)$ is the Bernstein polynomials of degree $m$.

A triangular Bézier patch is defined in the barycentric coordinates that is denoted $\mathbf{u}:=(u, v, w)$ with $u+v+w=1$. The expression is

$$
\mathbf{b}(\mathbf{u})=\sum_{|\mathbf{i}|=n} \mathbf{b}_{\mathbf{i}} B_{\mathbf{i}}^{n}(\mathbf{u}), \quad|\mathbf{i}|=i+j+k
$$

where $\mathbf{b}_{\mathbf{i}}$ is a triangular array of the control net and

$$
B_{\mathbf{i}}^{n}(\mathbf{u})=\left(\begin{array}{c}
n \\
\mathbf{i}
\end{array}\right) u^{i} v^{j} w^{k} ; \quad|\mathbf{i}|=n, \quad\left(\begin{array}{c}
n \\
\mathbf{i}
\end{array}\right)=\frac{n !}{i ! j ! k !}
$$

are the bivariate Bernstein polynomials. In this paper we use quartic Bézier patches thus degree $n$ is set to 4 .

The triangular spline surface used in this paper is represented by Bézier patches. Computing the necessary control points $\mathbf{b}_{\mathbf{i}}$ from the original mesh, we can obtain the corresponding Bézier patches. Composing all of them, the resulting surfaces is a $C^{2}$ surface if the original mesh is regular.

We utilize Sabin's rules [14 [15] to compute Bézier points. Let $P_{0}$ be the ordinary vertex and $P_{1}(i) ; i=1, \cdots, 6$ be the six neighboring vertices of $P_{0}$. In the case of quartic triangular Bézier patch there are fifteen control points, however, just four distinct rules exist due to symmetry. The following is the rules to compute Bézier control points $Q_{i j k}$ as illustrated in Fig. 1]:

$$
\begin{aligned}
& 24 Q_{400}=12 P_{0}+2 \sum_{i=1}^{6} P_{1}(i), \\
& 24 Q_{310}=12 P_{0}+3 P_{1}(1)+4 P_{1}(2)+3 P_{1}(3)+P_{1}(4)+P_{1}(6), \\
& 24 Q_{211}=10 P_{0}+6 P_{1}(1)+6 P_{1}(2)+P_{1}(6)+P_{1}(3), \\
& 24 Q_{220}=8 P_{0}+8 P_{1}(2)+4 P_{1}(1)+4 P_{3}(3) .
\end{aligned}
$$




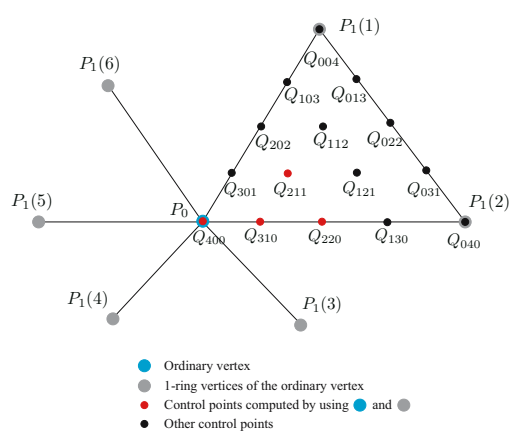

Fig. 1. Control points for triangular Bézier patch

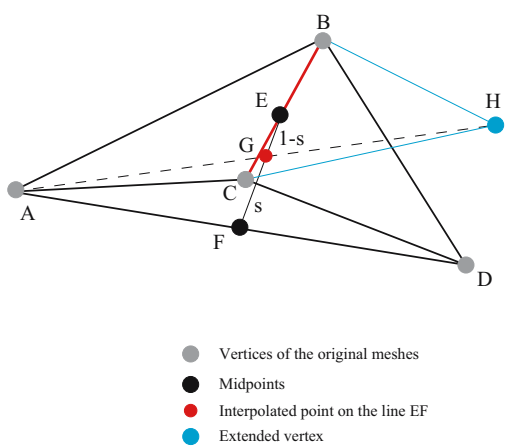

Fig. 2. Mesh extension and definition of parameter $s$

The remaining control points are obtained by applying the same rules to the neighboring vertices of $P_{1}(1)$ and $P_{1}(2)$.

\section{Mesh Extension}

In this section we briefly describe the method of mesh extension 12 to represent sharp features on a regular mesh. First, a designer specifies an edge to be a crease, then the meshes sharing the specified edge are separated. Next, we make extended vertices at the opposite side of the specified edge for both triangles. As shown in Fig. 2, let the original triangles be ABC and BCD. The edge BC is specified to create a crease. Point $\mathrm{E}$ is the midpoint of the edge $\mathrm{BC}$ and $\mathrm{F}$ is the midpoint of $\mathrm{AD}$. Then the position of $\mathrm{G}$ is parametrically defined so as to satisfy the relation

$$
G=s E+(1-s) F
$$

where $s$ denotes the parameter to control the sharpness of the crease. Finally the extended vertex $\mathrm{H}$ is defined as the position to satisfy $\mathrm{H}=2 \mathrm{AG}$ and the control triangle $\mathrm{BCH}$ is produced. In the opposite side, similar procedure is conducted with same parameter $s$.

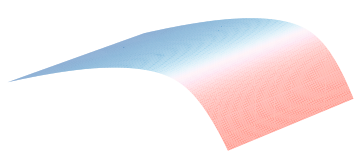

(a) $\mathrm{s}=0$ (smooth surface)

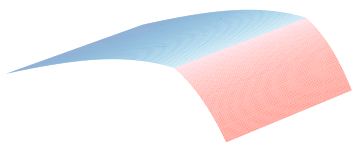

(b) $\mathrm{s}=0.5$ (crease)

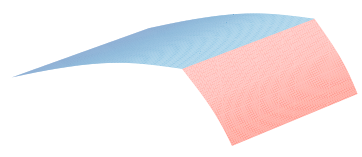

(c) $\mathrm{s}=1.0($ crease $)$

Fig. 3. Examples of generating crease edges for regular mesh 
Inputting $s$, all Bézier control points are determined and we get a regenerated surface where a sharp crease appears. By changing the value of $s$, the designer is able to control the sharpness of the crease. Fig. 3 shows examples of the surfaces created by this method. When $s$ is equal to 0 , the resulting surface is the original $C^{2}$ surface itself(Fig. 3(a)). When $s$ is equal to 1, the resulting surface(Fig. 3 (c)) is same as the shape of the subdivision crease. For the details of generating a dart or a corner, see 12 .

\section{Sharp Features on Non-regular Meshes}

This section introduces the rules to generate sharp features such as creases and corners on non-regular meshes. Higashi et al. [12] presented the method of mesh extension to create sharp features on regular meshes described in the previous section, however, computing rules for generating them on non-regular meshes are not shown. We have developed a method based on the mesh extension technique and Peters' algorithm [16] to generate a smooth surface with sharp features on a mesh including an extraordinary vertex.

\subsection{Creating a Crease}

There are numerous types of non-regular meshes. Here, non-regular meshes mean the set of meshes around an extraordinary vertex whose valance is not six. In this paper, we deal with the case of $n=5$ because the proposed method can be applied to other cases such as $n=4$ or 7 .

In the case of $n=5$, there are five meshes around an extraordinary vertex. Crease edges are defined on the two edges that divide these five meshes into two and three. On the side of the three meshes, the same rule is applied to obtain the control points as in the case of regular meshes. On the side of the two meshes, there are three steps to compute the control points.

In Fig. 4 (a), the input meshes are shown. For the first step of the process, as depicted in Fig. 4 (b), one edge is changed to connect the vertex in orange with the extraordinary vertex. Then, two meshes including crease edges are extended to make parallelograms using two new vertices which are colored red. By this treatment, the original non-regular meshes are now regular. Using ordinal Sabin's rules, control points represented by small red point are computed. Five white points are computed by Sabin's rules with one extended vertex and five original vertices. And small black control points are obtained by using original six vertices.

Second, the same process is conducted in the opposite side as shown in Fig. 4 (c). In the third step, as illustrated in Fig. 4 (d), the positions of two control points in yellow are modified to be middle between two adjacent control points. This calculation is done to keep $G^{1}$ continuity along the edge. Now, all of the required control points are available and we have the resulting surface by composing five Bézier patches that is expressed with Eq. (2). Note that the vertices of the extended meshes are only used to compute the necessary control 
(a)

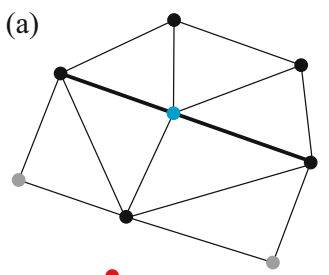

(c)

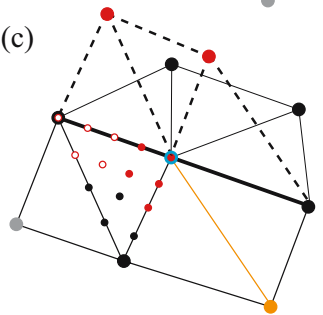

- Extraordinary vertex

- 1-ring ordinary vertices

- Adjacent vertices

Vertex used in step 1 and 2

- Vertex of extended mesh

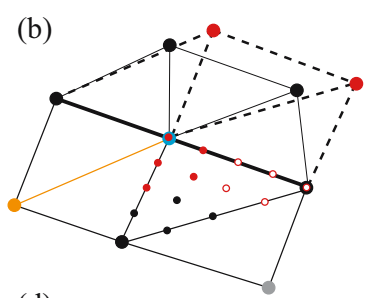

(d)

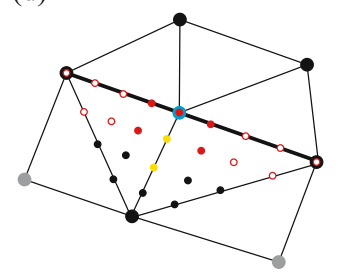

- Control points using Sabin' s rules with extended vertices

- Control points using orange vertices in step 1 and 2

- Control points using Sabin's rules with original vertices

- Control points to be modified for smoothness in step 3

Fig. 4. Description of the presented procedure: (a) the input meshes and vertices, (b) change of an edge(orange) and computation of control points(red) with extended meshes, (c) same procedure with (b) on the other side, (d) modification of the positions of the control points(yellow) to be smooth surface

points. This surface represents the case of $s=1$, therefore, the shape of the crease is identical to the original mesh. In order to represent the case of $s=0$, we adopt Peters's scheme [16] and these two surfaces are linearly interpolated with parameter $s$. Thus the surface changes its shape between these two shapes by inputting the parameter $s$.

\subsection{Creating a Corner}

To represent a sharp corner, we defined another rule. For simplicity, the case of $n=3$ is described. As shown in Fig. 5 there are three faces meeting at the target corner. Control points of each faces are obtained by using mesh extension procedure. Making parallelograms, five faces are generated as if the target corner vertex is the center of six meshes. Control points are calculated by Sabin's rules with these six vertices. Performing same procedure on the other two faces, all required control points are obtained. These control points represent the shape of the input mesh itself $(s=1)$. Then, using Peters' scheme [16] with the original vertices, we obtain control points to express a smooth surface $(s=0)$.

Seven control points, namely the corner point and its surrounding six points, are employed to represent a sharp corner. These points are colored blue in Fig. 6. Using the shape control parameter $s$, these seven control points are linearly interpolated as

$$
\boldsymbol{p}_{\text {new }}=(1-s) \boldsymbol{p}_{\text {smooth }}+s \boldsymbol{p}_{\text {sharp }}
$$




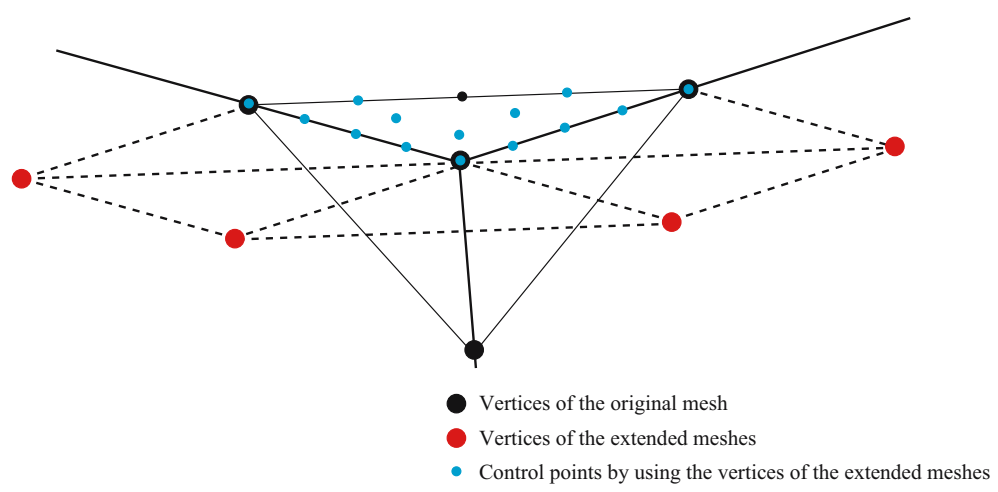

Fig. 5. An illustration of mesh extension to obtain control points for corner triangle faces. Starting from one of the corner mesh, four vertices are generated by making parallelogram. The same procedure is conducted on remaining two faces.

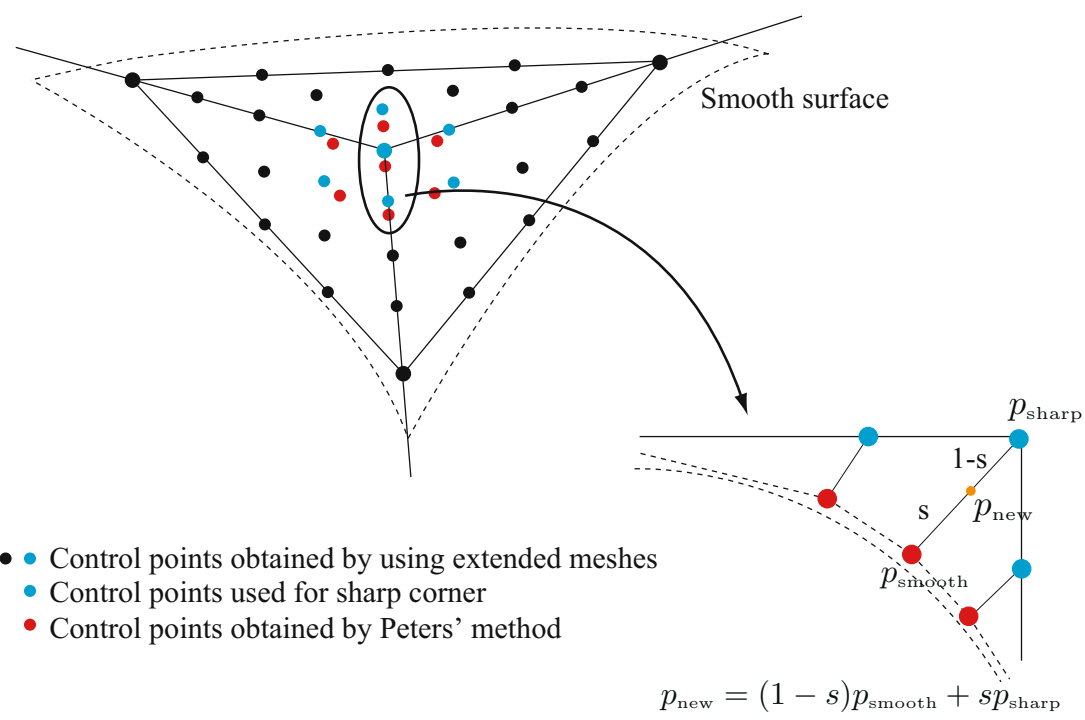

Fig. 6. Description of sharp corner generation. To change the shape of the corner, colored seven control points are used. These control points are linearly interpolated between Peters' control points (red) and control points (blue) obtained by using mesh extension.

where $\boldsymbol{p}_{\text {smooth }}$ is the position of the control points obtained by using Peters's scheme and $\boldsymbol{p}_{\text {sharp }}$ denotes the position of the control points generated by the mesh extension. Inputting some value to the parameter $s$, new control points $\boldsymbol{p}_{\text {new }}$ are computed by Eq. (9). By changing the value of the parameter $s$, we have a smooth corner $(s=0)$ and a sharp corner $(s=1)$. 


\section{Results}

This section provides application results where the presented method is used to represent sharp features on non-regular meshes. Here, results of generating creases are given in the case of valence $n=4,5$ and 7 . Tested meshes are depicted in Fig. 7 Figs. $8-10$ show results of each cases, where parameter $s$ is changed from 0 to 0.5 and 1 . When $s=0$, the resulting surface is identical to the smooth surface that is obtained by using the input mesh. On the other hand, if $s$ becomes greater than 0 , creases appear at the specified edges. When $s$ is equal to 1 , the shape of the crease is same as the shape of the input mesh. A small undulation is observed around the extraordinary vertex when $s=0.5$. The reason is that the smooth $\operatorname{surface}(s=0)$ is constructed by using Peters' scheme, where the tangential plane on the extraordinary vertex is arbitrarily input by a user. And the shapes of each Bézier patches are influenced by the tangential plane. Therefore, the crease line undulates when the mesh is not regular because tangent vectors are not necessarily parallel to the crease edges. This must be conquered to generate high quality creases.

Fig. 11] represents the case of sharp corner. From this picture, making a sharp corner is also successfully performed.

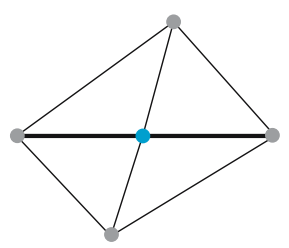

$\mathrm{n}=4$ (non-regular)

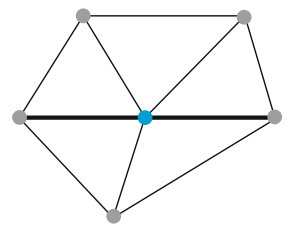

$\mathrm{n}=5$ (non-regular)

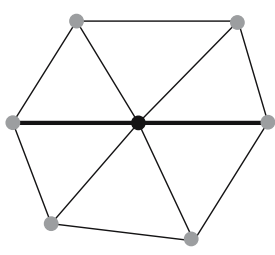

$\mathrm{n}=6$ (regular)

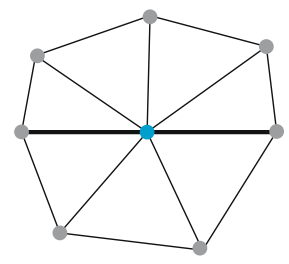

$\mathrm{n}=7$ (non-regular)

- Ordinary vertex( regular meshes)

- Extraordinary vertex( non-regular meshes)

- 1-ring vertices

Normal mesh edge

Crease edge

Fig. 7. Types of meshes used to produce examples

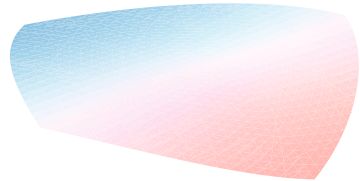

(a) $\mathrm{s}=0$ (smooth surface)

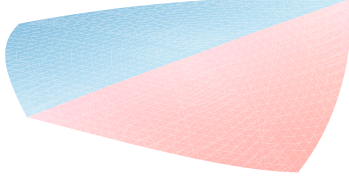

(b) $\mathrm{s}=0.5$ (crease)

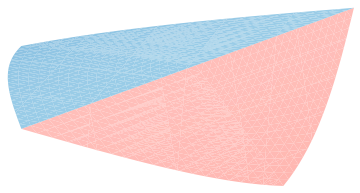

(c) $\mathrm{s}=1.0$ (crease)

Fig. 8. Results of generating creases in the case of $n=4$ 


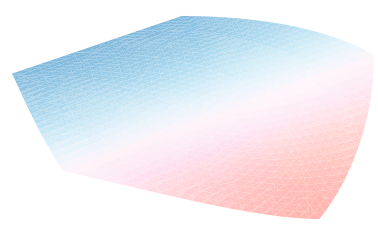

(a) $s=0$ (smooth surface)

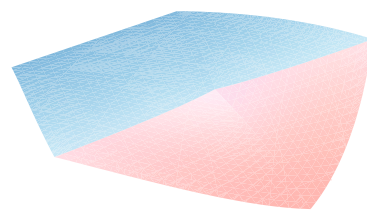

(b) $\mathrm{s}=0.5$ (crease)

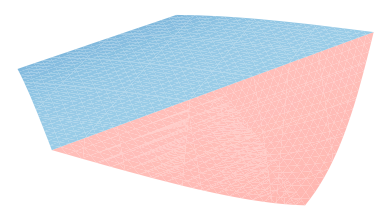

(c) $\mathrm{s}=1.0$ (crease)

Fig. 9. Results of generating creases in the case of $n=5$

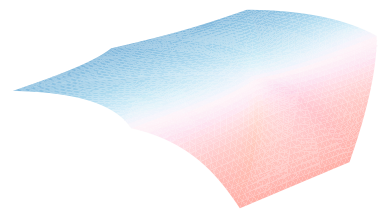

(a) $\mathrm{s}=0$ (smooth surface)

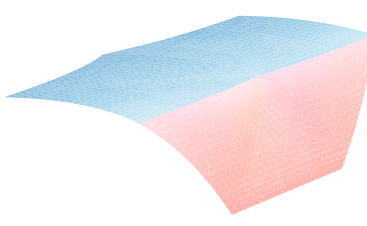

(b) $\mathrm{s}=0.5$ (crease)

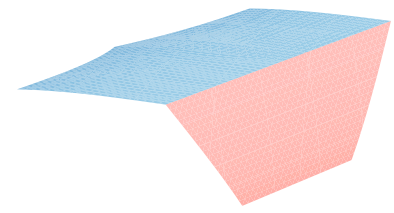

(c) $\mathrm{s}=1.0$ (crease)

Fig. 10. Results of generating creases in the case of $n=7$

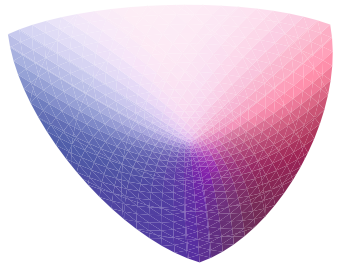

(a) $\mathrm{s}=0$ (smooth surface)

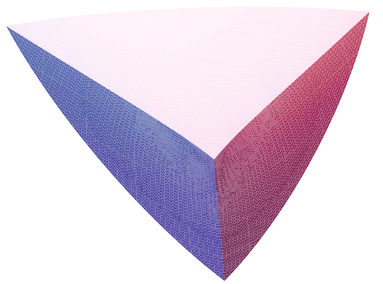

(b) $\mathrm{s}=0.5$ (corner)

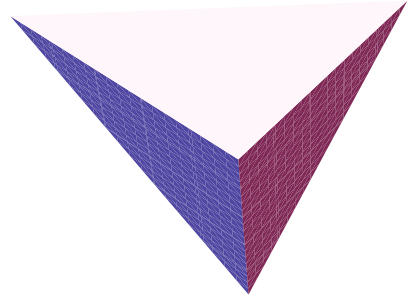

(c) $\mathrm{s}=1.0$ (corner)

Fig. 11. Results of generating sharp corner in the case of $n=3$

\section{Conclusion}

This paper presented a method to generate sharp features on non-regular meshes. Our method is based upon the regular version of the mesh extension technique and we have developed new schemes to deal with non-regular meshes. Results suggest that the method is effective for the tested cases. Future work would be an exploration for more various cases and a pursuit of the quality of features.

Acknowledgement. This study was financially supported by the High-tech Research Center for Space Robotics from the Ministry of Education, Sports, Culture, Science and Technology, Japan. 


\section{References}

1. Nasri, A.H.: Polyhedral Subdivision Methods for Free-Form Surfaces. ACM Transactions on Graphics 6(1), 29-73 (1987)

2. Hoppe, H., DeRose, T., Duchamp, T., Halstead, M.: Piecewise Smooth Surface Reconstruction. In: Proc. SIGGRAP 1994, pp. 295-302 (1994)

3. Loop, C.: Smooth Subdivision Surfaces Based on Triangles, Master's thesis, Department of Mathematics, University of Utah (1987)

4. DeRose, T., Kass, M., Truong, T.: Subdivision Surfaces in Character Animation. In: Proc. SIGGRAPH 1998, pp. 85-94 (1998)

5. Biermann, H., Levin, A., Zorin, D.: Piecewise Smooth Subdivision Surfaces with Normal Control. In: Proc. SIGGRAPH 2000, pp. 113-120 (2000)

6. Ying, L., Zorin, D.: Nonmanifold Subdivision. In: Proc. IEEE Visualization, pp. 325-332 (2001)

7. Sederberg, T.W., Zheng, J., Bakenov, A., Nasri, A.: T-splines and T-NURCCs. ACM Transactions on Graphics 22(3), 477-484 (2003)

8. Hahmann, S., Bonneau, G.-P.: Triangular $G^{1}$ interpolation by 4 -splitting domain triangles. Computer Aided Geometric Design 17, 731-757 (2000)

9. Hahmann, S., Bonneau, G.-P.: Polynomial Surfaces Interpolating Arbitrary Triangulations. IEEE Transactions on Visualization and Computer Graphics 9(1), 99-109 (2003)

10. Hahmann, S., Bonneau, G.-P.: Hierarchical Triangular Splines. ACM Transactions on Graphics 24(4), 1374-1391 (2005)

11. Loop, C.: Smooth Spline Surfaces over Irregular Meshes. In: Proc. SIGGRAP 1994, pp. 303-310 (1994)

12. Higashi, M., Inoue, H., Oya, T.: High-Quality Sharp Features in Triangular Meshes. Computer-Aided Design \& Applications 4, 227-234 (2007)

13. Farin, G.: Curves and Surfaces for CAGD, 5th edn. Academic Press, London (2005)

14. Boem, W.: The De Boor Algorithm for Triangular splines. In: Surfaces in Computer Aided Geometric Design, pp. 109-120. North-Holland, Amsterdam (1983)

15. Boem, W.: Generating the Bezier Points of Triangular Spline. In: Surfaces in Computer Aided Geometric Design, pp. 77-91. North-Holland Publishing Company, Amsterdam (1983)

16. Peters, J.: Smooth Patching of Refined Triangulations. ACM Transactions on Graphics 20(1), 1-9 (2001) 